\title{
A Newly Developed 1.2MV Field Emission Transmission Electron Microscope and Visualization of Topological Quantum Phenomena
}

\author{
Nobuyuki Osakabe \\ Central Research Laboratory, Hitachi, Ltd., Kokubunji, Tokyo
}

We have developed bright and monochromatic field-emission electron beams over 40 years and used them to study topological quantum phenomena by visualizing phase of electrons having wave-particle duality [Figure 1][1]. As it turns out, every time we developed a brighter electron beam, electron interference experiments became easier to perform and the precision in the phase measurements increased, thus opening up new application fields.

For example, we can now carry out fundamental experiments in quantum mechanics that were once regarded as thought experiments. Examples include conclusive experiments on the Aharonov-Bohm effect using toroidal ferromagnets completely shielded by superconductor [Figure 2][2]. The topology employed in the experiment also enabled us to observe the quantization of magnetic flux in units of $h / 2 e$ covered by superconducting wave function. This extends to the observation of quantized magnetic vortices introduced in the type-II superconductor when the magnetic field is applied [3]. The pinning mechanism of vortices has industrial importance for high-magnetic field application of superconductor such as MRI magnet and linear motor cars. Also, visualizing skyrmions as topological objects in $\mathrm{MnSi}$ by Lorentz microscopy have recently become possible [4].

In this talk, I present the development of an atomic-resolution holography electron microscope with an acceleration voltage of 1.2 MV [Figure 3] under the FIRST Tonomura project, as well as the historical review of electron holography with advances in coherence and brightness of electrons, since a bright electron source using field-emissions was the most decisive factor in the development of electron holography. The new technologies introduced in the 1.2-MV microscope will be described.

This microscope will be used to solve problems inhibiting innovation such as the origin of coercive force in rare-earth permanent magnet and dynamics of skyrmion driven by extremely small current.

This research was supported by the grant from the Japan Society for the Promotion of Science (JSPS) through the Funding Program for World-Leading Innovative R\&D on Science and Technology (FIRST Program)," initiated by the Council for Science and Technology Policy (CSTP) under the programs of "Development and Application of an Atomic-resolution Holography Electron Microscope" and "Quantum Science on Strong Correlation".

\section{References:}

[1] A. Tonomura et al., Amer. J. Phys., 57 (1989r) p. 117.

[2] N. Osakabe et al., Phys. Rev. A 34 (1986) p. 815.

[3] K. Harada et al., Nature 360 (1992) p. 51.

[4] A. Tonomura et al.,Nano Letters 12 (2012) p. 1673. 

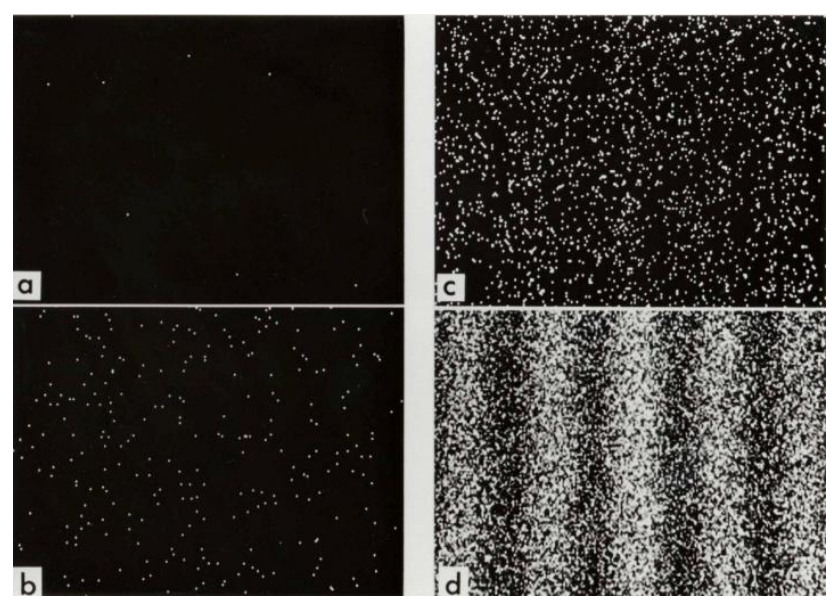

Figure 1. Build-up of an electron interference pattern. Numbers of electrons are (a) 10, (b) 100, (c) 3,000 , (d) 70,000.

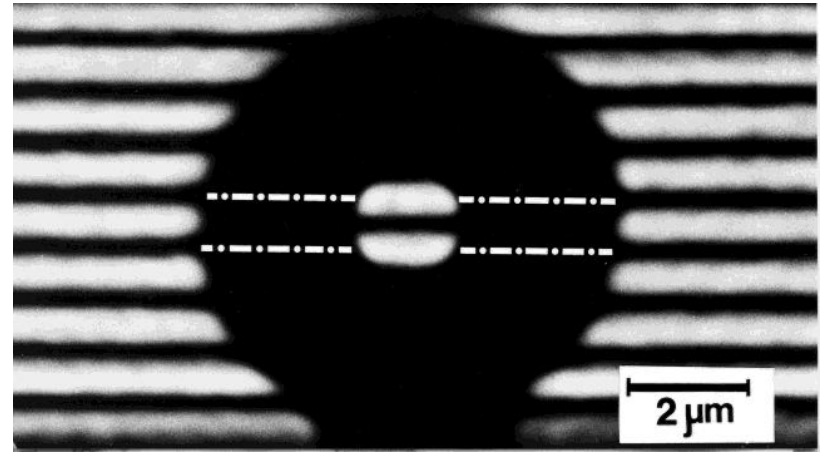

Figure 2. The Aharonov-Bohm (AB) effect.

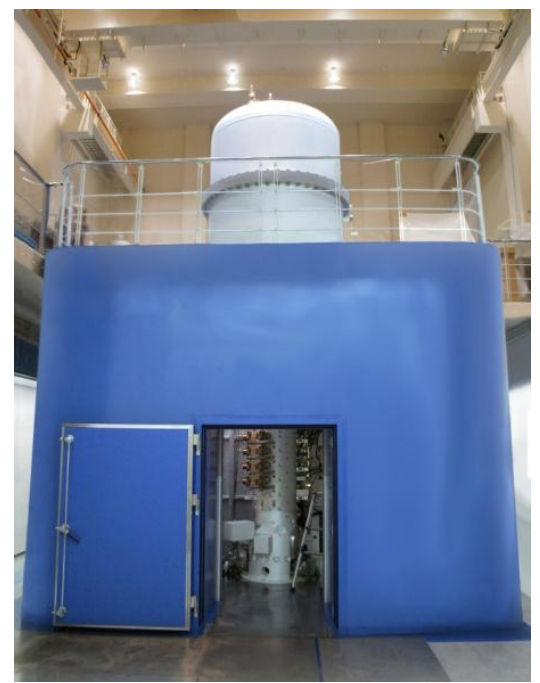

Figure 3. 1.2-MV field-emission holography electron microscope 